\title{
A TWO-SIDED SHOOTING METHOD IN COMPUTATION OF TRAVELLING COMBUSTION WAVES OF A SOLID MATERIAL
}

\author{
ANDONOWATI ${ }^{1}$ \\ (Received 11 July 1994; revised January 1995)
}

\begin{abstract}
Numerical solutions for travelling combustion waves of a solid material are sought. The algorithm of computation is based on a two-sided shooting method. It is found that there is a lower bound of the wave speed $c$, say $c^{*}$, such that for $c<c^{*}$ no numerical solution can be constructed. This $c^{*}$ is a function of the activation energy of the medium.
\end{abstract}

\section{Introduction}

A simple model for the combustion of a solid material is given by

$$
\begin{aligned}
\frac{\partial \theta}{\partial t} & =\nabla^{2} \theta+H \chi f(\theta), \\
\frac{\partial \chi}{\partial t} & =\nabla^{2} \chi-\epsilon \chi f(\theta), \\
\text { with } f(\theta) & =\exp \left\{\frac{\alpha \theta}{\alpha+\theta}\right\},
\end{aligned}
$$

where $\theta$ and $\chi$ are the temperature and the concentration of the combustible material, and $\mathbf{x}$ and $t$ are independent variables for space and time. $H$ is a positive number related to the chemical properties of the combustible material, the external temperature, and the geometrical dimension of the medium. The parameter $\alpha$, considerably larger than one, is proportional to the activation energy of the medium and $\epsilon=e^{-\alpha}$.

The governing equations for the travelling combustion wave for the above problem may be obtained by letting $\mathbf{x}=x$, where $-\infty<x<\infty$ and $\xi=x+c t$, where $c$ is

\footnotetext{
'Department of Mathematics and Statistics, McGill University, 805 Sherbrooke Street West, Montreal, Québec, Canada, H3A 2K6.

(C) Australian Mathematical Society, 1996, Serial-fee code 0334-2700/96
} 
the wave speed. Equations (1) and (2) become

$$
\begin{aligned}
\theta^{\prime \prime}-c \theta^{\prime}+H \chi f(\theta) & =0 \\
\chi^{\prime \prime}-c \chi^{\prime}-\epsilon \chi f(\theta) & =0
\end{aligned}
$$

where the prime denotes the derivative with respect to $\xi$. The relevant boundary conditions to the problem are

$$
\theta(-\infty)=0, \quad \theta(\infty)=\theta_{\max }, \quad \chi(-\infty)=1, \quad \chi(\infty)=0,
$$

where $\theta_{\max }$ is the maximum temperature reached after combustion, whose value has to be determined. It should be noted that no solution to (4) and (5) subject to the boundary conditions (6) is possible. This can be seen by taking the limit as $\xi \rightarrow-\infty$ in (4); the left hand side of this equation gives 1 , while the right hand side equals 0 .

In [2], Tam replaced the function $f(\theta)$ by

$$
g(\theta)= \begin{cases}\exp \{\alpha \theta /(\alpha+\theta)\}-1 & \text { if } \theta \geq 0 \\ 0 & \text { otherwise }\end{cases}
$$

By assuming that $\psi=\epsilon \theta+H \chi$ is bounded and satisfies the boundary conditions imposed on $\theta$ and $\chi$, we have $H=\epsilon \theta+H \chi$ and $\theta_{\max }=H / \epsilon$. Thus, instead of using (4), (5), and the boundary conditions above, Tam in [2] considered

$$
\theta^{\prime \prime}-c \theta^{\prime}+(H-\epsilon \theta) g(\theta)=0
$$

subject to boundary conditions

$$
\theta(-\infty)=0, \quad \theta(\infty)=H / \epsilon
$$

Tam then proved the existence of solutions using a phase-plane method. Previously, a number of authors proved the existence of solution for similar problems. Aronson and Weinberger [1], for example, gave a detailed proof of the existence of solution for a more general function of $F(\theta)$ such that $\theta$ satisfies the equation

$$
\theta^{\prime \prime}-c \theta^{\prime}+F(\theta)=0
$$

subject to conditions $\theta(\xi) \in[0,1], \theta(\xi) \neq 0$, and $\lim _{\xi \rightarrow \infty} \theta(\xi)=0$. Also see [5] for a discussion of such a problem.

The algorithm of computation is based on a shooting method as follows. Since the problem is invariant under translation we may choose the location of $\xi=0$ to be such that $\theta(0)=H / \epsilon-\delta$, for a small positive $\delta$. For each $c$, we derive an a priori bound for $\theta^{\prime}(0)$. Assuming the solution exists for some $c$; for those $c$, one can choose 
a set of values of $\theta^{\prime}(0)$ such that the solution can be extended to the right to satisfy the boundary condition at $\xi=\infty$. Furthermore, there must be a subset of such values such that the solution can be extended to the left to satisfy the boundary condition at $\xi=-\infty$. Indeed in [2], Tam showed analytically that for $c \geq \sqrt{4 \bar{\sigma}}$, where $\bar{\sigma}=\max _{\theta}\{F(\theta) / \theta\}$, the solution to (8) and (9) exists. This method of computation was motivated by the existence proof in [3]. A similar method was employed in [4] by Tam and Andonowati for a different problem.

In the next section we present the properties of solutions $\theta(\xi)$. We derive an $a$ priori bound for $\theta^{\prime}(0)$ in Section 3. The algorithm of computation is then constructed. In Section 4 numerical results are obtained; and concluding remarks are presented in Section 5 .

\section{Behaviour of solutions}

We note that the differential equation (8) above has two critical points $\left(\theta, \theta^{\prime}\right)=$ $(0,0)$ and $\left(\theta, \theta^{\prime}\right)=(H / \epsilon, 0)$. By linearizing the equation near the critical points, we should have $c^{2} \geq 4 H$ in order to satisfy the boundary condition at $\xi=-\infty$. When $c^{2} \geq 4 H$ the critical point $(0,0)$ is a stable node while the critical point $(H / \epsilon, 0)$ is an unstable node.

The following properties of $\theta$ can be derived easily from (8) and (9) or by examining the direction field of the phase plane $\theta^{\prime} \mathrm{vs} \theta$. These properties of the solution $\theta$ are to be used in constructing the algorithm of computation in the next section.

PROPERTY 1. If $\theta(\xi)$ is a solution of (8) with the boundary conditions (9) then $0<$ $\theta(\xi)<H / \epsilon$ and $\theta(\xi)$ is monotonically increasing.

PROPERTY 2. Let $F(\theta(\xi))=(H-\epsilon \theta(\xi)) g(\theta(\xi))$, then $F(\theta(\xi))$ has exactly one extremum which is a relative maximum, say, at $\xi=\xi_{m}$. For $\xi<\xi_{m}, F(\theta(\xi))$ is monotonically increasing and for $\xi>\xi_{m}, F(\theta(\xi))$ is monotonically decreasing.

PROPERTY 3. $\theta(\xi))$ has exactly one inflection point, $\xi_{e}$, such that for $\xi<\xi_{e}, \theta^{\prime}(\xi)$ is monotonically increasing and for $\xi>\xi_{e}, \theta^{\prime}(\xi)$ is monotonically decreasing.

PROPERTY 4. If $\xi_{e}$ is the inflection point of $\theta(\xi)$ and $\xi_{m}$ is the point such that $F\left(\theta\left(\xi_{m}\right)\right)$ is a maximum then $\xi_{e} \leq \xi_{m}$.

\section{An algorithm based on an a priori bound}

For fixed numbers $\alpha$ and $H$, let $\delta$ be a small positive number relative to $\theta_{\max }=H / \epsilon$ such that $H / \epsilon-\delta>\theta_{m}$, where $F\left(\theta_{m}\right)=\left(H-\epsilon \theta_{m}\right) g\left(\theta_{m}\right)$ is the maximum. Such $\theta_{m}$ 
satisfies $F^{\prime}\left(\theta_{m}\right)=0$ or

$$
\begin{gathered}
\left(H-\epsilon \theta_{m}\right)\left(\frac{\alpha}{\alpha+\theta_{m}}\right)^{2} \exp \left\{\alpha \theta_{m} /\left(\alpha+\theta_{m}\right)\right\}=\epsilon\left(\exp \left\{\alpha \theta_{m} /\left(\alpha+\theta_{m}\right)\right\}-1\right) \\
0<\theta_{m}<H / \epsilon
\end{gathered}
$$

We choose $\xi=0$ to be a point such that

$$
\theta(0)=H / \epsilon-\delta>\theta_{m}
$$

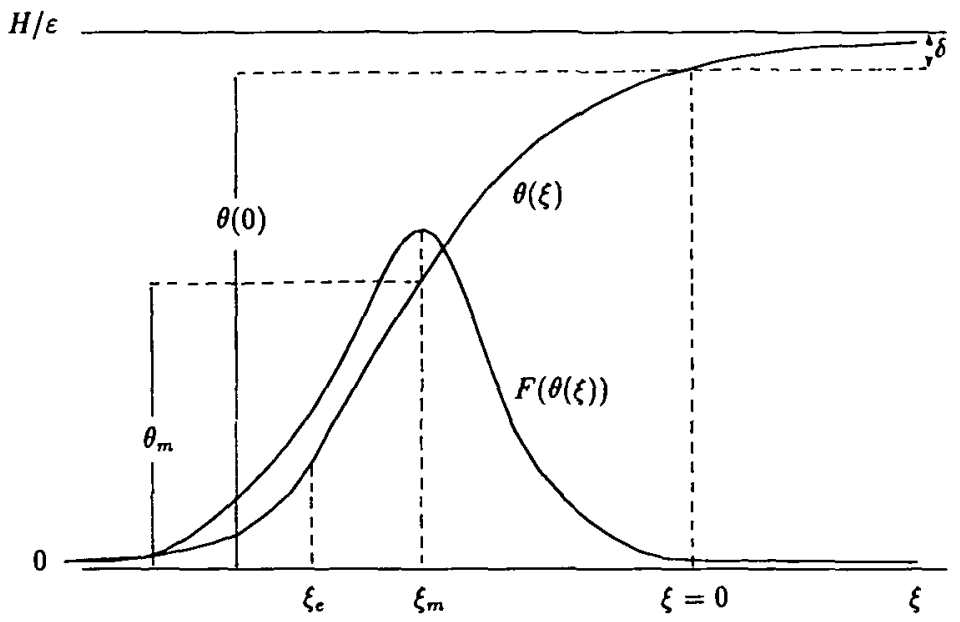

FIGURE 1. $\theta(\xi)$ and $F(\theta(\xi))$ vs $\xi$. The choice of $\delta$ in the algorithm is such that $H / \epsilon-\delta>\theta_{m}$.

Given $\theta(0)=H / \epsilon-\delta$, an a priori bound for $\theta^{\prime}(0)$ can be obtained as follows. From (8)

$$
\theta^{\prime}(0)=\frac{\theta^{\prime \prime}(0)}{c}+\frac{1}{c}\{H-\epsilon \theta(0)\} g(\theta(0)) .
$$

Using Properties 1 and 4, we have $\theta(0)>\theta\left(\xi_{m}\right) \geq \theta\left(\xi_{e}\right)$, where $F\left(\theta\left(\xi_{m}\right)\right.$ ) is the maximum and $\xi_{e}$ is the inflection point of $\theta(\xi)$. From Property 3 , we conclude that $\theta^{\prime \prime}(0)<0$ (see Figure 1). Thus,

$$
0<\theta^{\prime}(0)<\frac{1}{c}\{H-\epsilon \theta(0)\} g(\theta(0)) .
$$

Substituting $\theta(0)=H / \epsilon-\delta$ into (14) and knowing that $g(\theta(0))=\exp (\alpha \theta(0) /(\alpha+$ $\theta(0))\}-1<e^{\alpha}=1 / \epsilon$, we have

$$
0<\theta^{\prime}(0)<\delta / c
$$


Further notice that we choose $\xi=0$ to be a point such that $\theta(0)$ is close to $H / \epsilon$. The reason for doing this is that the critical point $(H / \epsilon, 0)$ is unstable. Thus the integration to the right of (8), using Runge-Kutta of order four, will accumulate a high truncation error for a reasonably long distance of $\xi$ from the initial point. The critical point $(0,0)$, on the other hand, is stable and so the truncation error of the integration to the left will not be multiplied at each step of integration.

The algorithm of computation is as follows. Since the solution of (8) exists only for $c \geq \sqrt{4 H}$, start with some fixed $c>\sqrt{4 H}$ and calculate $\delta / c$. As the critical point $(H / \epsilon, 0)$ is unstable and $(0,0)$ is stable, integrate $(8)$ to the right with $\theta(0)=H / \epsilon-\delta$ and a fixed $\theta^{\prime}(0) \in(0, \delta / c)$. Allow the value of $\theta^{\prime}(0)$ to change within the bound $0<\theta^{\prime}(0)<\delta / c$, until we find that the integration to the right can go to, say $L_{1}$, which $\theta(\xi)$ behaves as predicted by its analytical properties, namely, $\theta(\xi)$ is monotonically increasing to $H / \epsilon$ and $\theta^{\prime}(\xi)$ is monotonically decreasing to 0 . Change $c$ and repeat the procedure to find the corresponding $\theta^{\prime}(0)$. Thus for each $\alpha$, we obtain a set

$$
\begin{aligned}
\Lambda_{\alpha}= & \left\{\left(c, \theta^{\prime}(0)\right) \mid \theta(0)=H / \epsilon-\delta \text { such that the integration of }(8)\right. \\
& \text { to the right has } \theta(\xi) \text { monotonically increasing to } H / \epsilon \text { and } \theta^{\prime}(\xi) \text { is } \\
& \text { monotonically decreasing to } 0\} .
\end{aligned}
$$

For each $\left(c, \theta^{\prime}(0)\right) \in \Lambda_{\alpha}$, we integrate (8) to the left to verify that $\theta(\xi)$ tends to zero.

It should be noted that the algorithm is contructed merely based on an a priori bound of the initial condition and the analytical properties of the solutions.

\section{Numerical results}

We demonstrate the above algorithm with $\alpha=10$ and $H=1.0$. Calculating $\theta_{\max }=H / \epsilon=H e^{\alpha}$ and $\theta_{m}$ from (11) we obtain $\theta_{\max }=22026.46579481$ and $\theta_{m}=$ 1425.31026371. By choosing $\delta=26.46579481$, we have $\theta(0)=22000.0>\theta_{m}$. As an example, let $c=16$. This gives $\delta / c=1.65411218$ and so $0<\theta^{\prime}(0)<$ 1.65411218. We integrate (8) to the right with $\theta^{\prime}(0)=0.1 N_{1}$, where $N_{1}$ runs from 1 to 16 and find that $\theta^{\prime}(0)=1.6$ is the best candidate for a refinement. We next integrate (8) to the right with $\theta^{\prime}(0)=1.6+0.01 N_{2}$, where $N_{2}$ runs from -10 to 10 and find $\theta^{\prime}(0)=1.64$ is the candidate for further refinement. The proccess continues until we obtain $\theta^{\prime}(0)=1.64018585$, where the integration to the right can go as far as $\xi=26.0$ in which $\theta(\xi)$ is monotonically increasing toward $\theta_{\max }=22026.46579481$ while $\theta^{\prime}(\xi)$ is monotonically decreasing to 0 . For different values of $c$ we repeat the process to find the corresponding $\theta^{\prime}(0)$. From the above procedure, we obtain $\Lambda_{\alpha=10}=$ $\left\{\left(c, \theta^{\prime}(0)\right) \mid \theta(0)=22000.0\right\}$. For each value of $\left(c, \theta^{\prime}(0)\right) \in \Lambda_{\alpha=10}$, we then integrate (8) to the left to check whether that value yields a solution. In the case of $c=16$, we 
obtain $\theta^{\prime}(0)=1.64018585$ and the integration to the left can go as far as $\xi=-200.0$, where $\theta(-200.0)=0.00472871$. We consider this value of $\theta(-200.0)$ is close to 0 , and thus a numerical solution for $\alpha=10, H=1.0$, and $c=16$ is established. We repeat the procedure for different values of $\alpha$.

It is found that as $c$ decreases, the integration to the left toward the stable node becomes increasingly difficult. It is then conceivable that there is a limit for $c$, say $c=c^{*}$, below which no travelling wave solution can be constructed. This $c^{*}$ is an increasing function of $\alpha$. We note that the parameter $\alpha$ is proportional to the activation energy of the material. Larger values of $\alpha$ correspond to more combustible material, and result in larger critical values $c^{*}$. Numerical results give a strong indication that solutions of (8) subject to the boundary conditions (9) exist for $c \geq c^{*}(\alpha)$. Thus it is suggested, numerically, that $c^{*}$ is the minimum speed for the combustion waves.

For $\alpha=10.0$ and $H=1.0$, Figure 2 shows the solution $\theta(\xi, c)$ for some $c$, $c>c^{*}(10)$. The numerical solution $\theta(\xi, c)$ for $\alpha=20.0$ is presented in Figure 3.

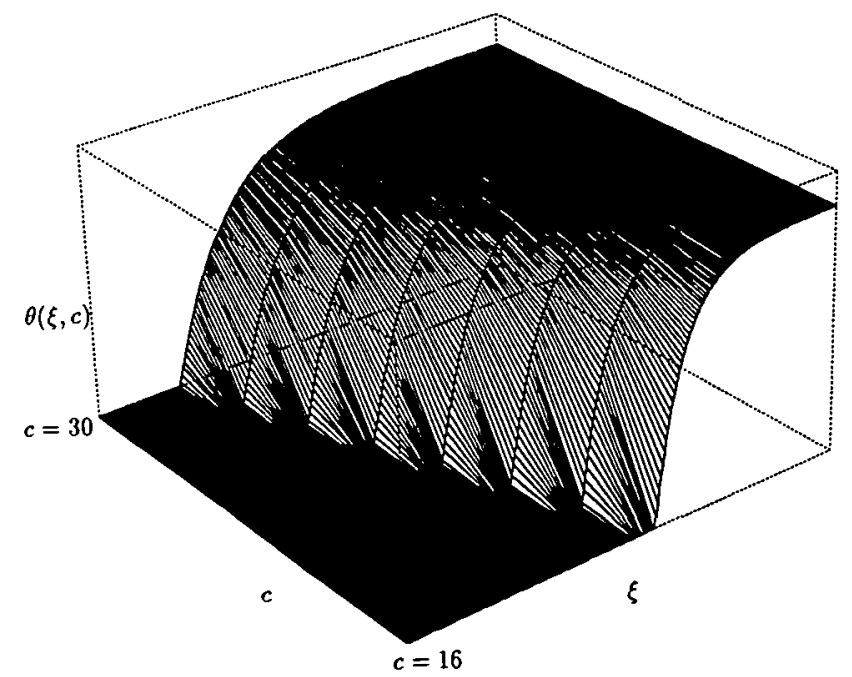

FIGURE 2. The solution $\theta$ for $\alpha=10.0,16 \leq c \leq 30$, with $H=1.0$. Note that $c^{*}(10)=13.4$.

We noted that in [2] Tam derived a sufficiency condition for the solution to exist, that is

$$
c \geq \sqrt{4 \bar{\sigma}}
$$

where

$$
\bar{\sigma}=\max _{\xi} \frac{F(\theta(c, \xi))}{\theta(c, \xi)}=\max _{0<\theta<H / \epsilon} \frac{F(\theta)}{\theta}
$$




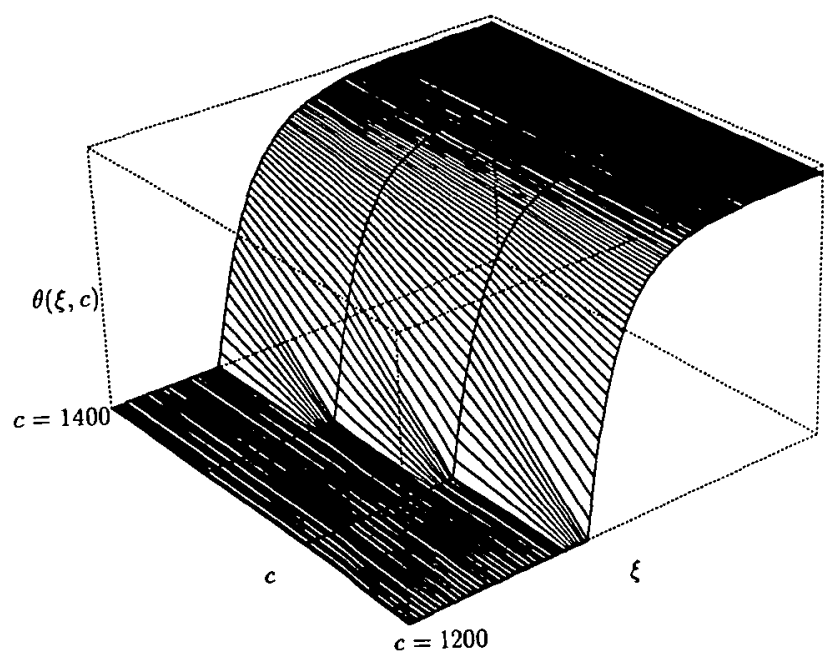

FIGURE 3. The solution $\theta$ for $\alpha=20.0,1200 \leq c \leq 1400$, with $H=1.0$. Note that $c^{*}(20)=910.0$.

and

$$
F(\theta(\xi, c))=(H-\epsilon \theta(\xi, c)) g(\theta(\xi, c))
$$

(see Figure 4). Let

$$
c^{s}(\alpha)=\sqrt{4 \bar{\sigma}}
$$

We present in Table 1 a comparison of the values of $c^{s}(\alpha)$ and $c^{*}(\alpha)$ for some $\alpha$. We find that $c^{s}(\alpha)$ is considerably larger than $c^{*}(\alpha)$ and thus the numerical result gives a better lower bound of $c$ for the solution to exist than the one found analytically in [2].

TABLE 1. The comparison of lower bounds for the wave speed $c$ derived analytically, $c^{5}$, and calculated numerically, $c^{*}$, for different values of $\alpha$ with $H=1.0$.

\begin{tabular}{||crrr||}
\hline \hline$\alpha$ & \multicolumn{1}{c}{$\bar{\sigma}$} & \multicolumn{1}{c}{$c^{s}$} & \multicolumn{1}{c|}{$c^{*}$} \\
\hline 10.0 & 90.629 & 19.0399 & 13.4 \\
\hline 12.0 & 455.597 & 42.6894 & 30.4 \\
\hline 14.0 & 2438.343 & 98.7592 & 70.8 \\
\hline 20.0 & 470302.493 & 1371.5721 & 910.0 \\
\hline \hline
\end{tabular}




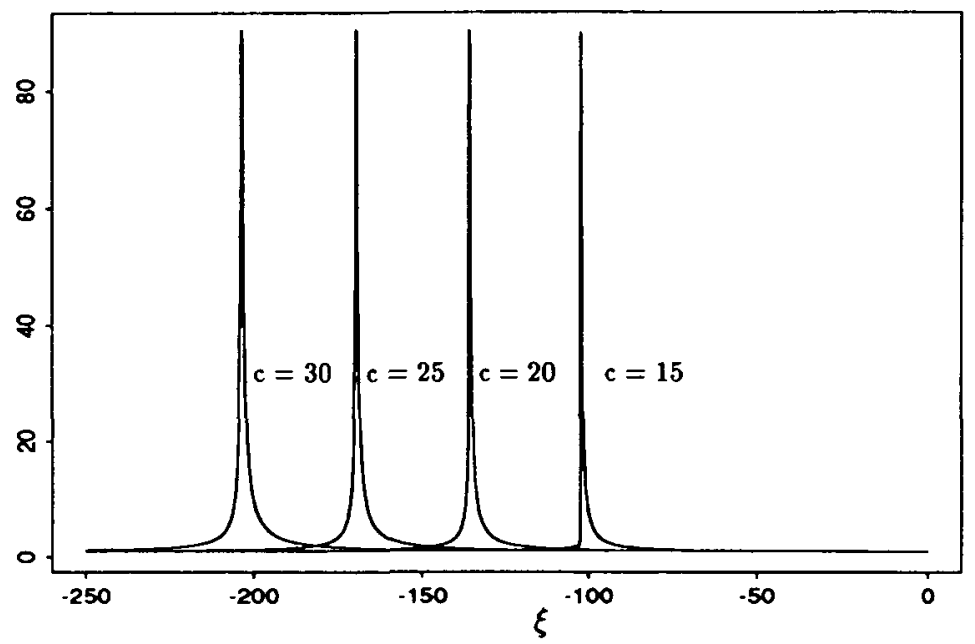

Figure 4. $F(\theta(c, \xi)) / \theta(c, \xi)$ vs $\xi$ for different $c: c=15,20,25$ and 30 , where $H=1.0$ and $\alpha=10.0$.

\section{Concluding remarks}

We have sought numerical solutions for travelling combustion waves of a solid material. The mathematical model is presented in (8) subject to the boundary conditions (9). Since the boundary conditions are prescribed at $\xi= \pm \infty$, to find a numerical solution for this problem we need to ask from which initial value of $\xi$ should an integration be executed. We answer the question by presenting a computational algorithm based on a two-sided shooting method.

Using this method, we establish a lower bound of $c$, say $c^{*}$, so that a solution should exist. This $c^{*}$ is a function of the activation energy $\alpha$ of the medium, and it increases as $\alpha$ increases. The numerical results also suggest that for a given set of parameters $\alpha$ and $H$ with $c \geq c^{*}$, the solution to the system is unique.

\section{Acknowledgment}

The author is very grateful to Prof. K. K. Tam for detailed comments and corrections on the manuscript of this paper. She would like to thank the referee for mentioning some errors on the manuscript and giving some useful suggestions. 


\section{References}

[1] D. G. Aronson and H. F. Weinberger, "Multidimensional nonlinear diffusion arising in population genetics", Advances in Mathematics 30 (1978) 33-76.

[2] K. K. Tam, "Traveling wave solutions for a combustion problem", Studies in Appl. Math. 81 (1989) 117-124.

[3] K. K. Tam, "Traveling wave solutions for combustion in a porous medium", Studies in Appl. Math. 81 (1989) 249-263.

[4] K. K. Tam and Andonowati, "Computation of travelling combustion waves in a porous medium", Studies in Appl. Math. 91 (1994) 179-187.

[5] Ya. B. Zeldovich, G. I. Barenblatt, V. B. Librovich and G. M. Makhiviladze, The mathematical theory of combustion and explosions (Consultants Bureau, Plenum, New York, 1985). 